\title{
Phase III trial results for tofacitinib bring new oral DMARD therapy a step closer for patients with rheumatoid arthritis
}

T wo phase III trials of the Janus kinase (JAK) inhibitor tofacitinib have produced favourable results in patients with active rheumatoid arthritis (RA). The studies, which were funded by Pfizer, the manufacturer of tofacitinib, and published in the New England Journal of Medicine, assessed the efficacy and safety of the drug as monotherapy versus placebo in patients for whom other DMARDs had failed, and as an alternative to adalimumab in combination with methotrexate in patients with an incomplete response to methotrexate.

Tofacitinib blocks transduction of signals from several interleukins by inhibiting receptors associated with JAK1 and JAK3, and thus interferes with lymphocyte functions. Phase II trials of the drug were encouraging, although its precise mode of action in RA remains unclear.

Roy Fleischmann of the Metroplex Clinical Research Center in Dallas, USA, an investigator involved in both of the published phase III studies, described the two key findings. Firstly, "tofacitinib is effective as monotherapy and may well not need to be given with methotrexate," he says. Methotrexate is intolerable for many patients, Fleischmann explains, yet "most biologic DMARDs require methotrexate to demonstrate their maximum efficacy - tofacitinib may not." The second key result is that "both doses of tofacitinib are as efficacious as adalimumab when they are given with methotrexate."

611 patients with active RA who had discontinued all DMARDs on account of lack of efficacy or adverse events were enrolled in the 6-month monotherapy trial. RA had been diagnosed using the American College of Rheumatology (ACR) 1987 revised criteria, and active disease was defined as $\geq 6$ tender or painful joints and $\geq 6$ swollen joints, out of 68 and 66 examined sites, respectively. Participants also had elevated erythrocyte sedimentation rates (ESR) or C-reactive protein levels, or both.
Patients randomized to the tofacitinib groups received two twice-daily doses of tofacitinib, either $5 \mathrm{mg}$ or $10 \mathrm{mg}$, for 6 months. In the placebo groups, patients were switched to one of the tofacitinib doses after 3 months. Primary efficacy end points were assessed at month 3 , consisting of the percentage of patients reaching an ACR20 response (an improvement from baseline of at least $20 \%$ in the number of swollen and tender joints as well as in at least three other ACR core set measures), an improvement in physical function (measured by the Health Assessment Questionnaire-Disability Index, HAQ-DI), and a Disease Activity Score for 28-joint counts based on ESR (DAS28-4ESR) of $<2.6$ (a score $<3.2$ using this composite measure indicates low disease activity).

Significant increases in the ACR20 rate and HAQ-DI scores were found for both doses of tofacitinib at 3 months in comparison with placebo, and patients who switched from placebo at the 3-month mark had similar rates of response at month 6 as were seen in patients in the treatment arms at month 3. Clinically meaningful improvements in fatigue levels were also found with tofacitnib versus placebo, but changes in DAS28-4ESR were not significant.

The second, 12-month trial enrolled 717 patients with active RA (by the same criteria as the first study) who were receiving stable doses of methotrexate but had an incomplete response to the drug. The same $5 \mathrm{mg}$ or $10 \mathrm{mg}$ twice-daily doses of tofacitinib were used as in the first trial, but were compared with either subcutaneous adalimumab (once every 2 weeks), or with placebo for 3 or 6 months followed by tofacitinib for the remainder of the trial. Adalimumab was included to enable estimation of the the relative safety and efficacy of tofacitinib, though a formal noninferiority comparison was not undertaken.

ACR20 rates, HAQ-DI scores and DAS28-4ESR were considerably better

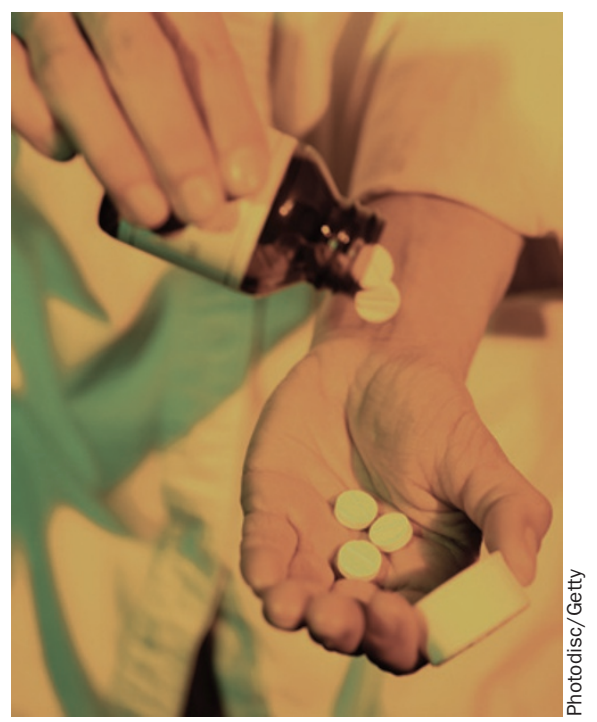

with active treatment at month 6 in comparison with placebo. ACR20 response rates for tofacitinib $5 \mathrm{mg}$ and $10 \mathrm{mg}(51.5 \%$ and $52.6 \%)$ were similar to that for adalimumab (47.2\%).

Serious adverse events were more frequent with tofacitinib than with placebo in both trials, and larger-scale, longerterm studies are ongoing to assess the increased risk. Furthermore, levels of LDL cholesterol were increased with tofacitinib, an effect also seen in phase II studies but not yet fully understood.

Tofacitinib is an oral drug and patients are likely to prefer it to parenteral biologic agents. Referring to the next stage of tests, Fleischmann adds, "if tofacitinib is clinically more effective and inhibits radiographic progression better than methotrexate, it should be a very viable alternative for patients."

Emma Leah

Original articles Fleischmann, R. et al. Placebocontrolled trial of tofacitinib monotherapy in rheumatoid arthritis. N. Engl. J. Med. 367, 495-507 (2012) | van Vollenhoven, R. F. et al. Tofacitinib or adalimumab versus placebo in rheumatoid arthritis. N. Engl. J. Med. 367 , 508-519 (2012) 